\title{
Acknowledgement to Reviewers of Metals in 2016
}

\author{
Metals Editorial Office \\ Published: 11 January 2017 \\ MDPI AG, St. Alban-Anlage 66, 4052 Basel, Switzerland; metals@mdpi.com
}

The editors of Metals would like to express their sincere gratitude to the following reviewers for assessing manuscripts in 2016.

We greatly appreciate the contribution of expert reviewers, which is crucial to the journal's editorial process. We aim to recognize reviewer contributions through several mechanisms, of which the annual publication of reviewer names is one. Reviewers receive a voucher entitling them to a discount on their next MDPI publication and can download a certificate of recognition directly from our submission system. Additionally, reviewers can sign up to the service Publons (https://publons.com) to receive recognition. Of course, in these initiatives we are careful not to compromise reviewer confidentiality. Many reviewers see their work as a voluntary and often unseen part of their role as researchers. We are grateful to the time reviewers donate to our journals and the contribution they make.

If you are interested in becoming a reviewer for Metals, see the link at the bottom of the webpage http://www.mdpi.com/reviewers.

The following reviewed for Metals in 2016:

Abadias, Gregory
Aboulkhair, Nesma T.
Adachi, Hiroki
Aghion, Eli
Aguey-Zinsou, Kondo-Francois
Ahmed, Essam
Ahmed, Hesham M.
Ahn, Byungmin
Ahn, Sangjoon
Ahn, Seokyoung
Aidun, Daryush
Akhiani, Hamed
Akiyama, Eiji
Alam, Shahria
Allard, Bénédicte
Allison, Paul G.
AlMangour, Bandar
Alorro, Richard Diaz
Al-Shamma'a, Ahmed
Álvarez-Ayuso, Esther
Alves, Alexandra
Amini, Ghobad
Alation

Amirthalingam, Murugaiyan
Anderson, Peter
Andersson, Jon M.
Angelastro, Andrea
Angelini, Emma
Anthony, Edward J.
Antipas, Georgios S. E.
Antonio, Faba
Antrekowitsch, Jürgen
Apreutesei, Mihai
Arenas, Maria Angeles
Argüello, José M.
Arslan, Zikri
Ashcroft, Ian
Astana, Rajiv
Astarita, Antonello
Atwater, Mark A.
Auinger, Michael
Austin, Rachel
Awual, Md. Rabiul
Azar, Amin S.
Baba, Koumei
Anto

Bacca, Mattia

Bachmaier, Andrea

Bagherifard, Sara

Balan, Tudor

Balandraud, Xavier

Bankiewicz, Dorota

Barbaro, Frank

Barbosa, J.

Bariani, Paolo F.

Barna, Martin

Barroi, A.

Barry, Carol

Bastidas, David M.

Batis, George

Bayraktar, Emin

Beck, Tilmann

Bellezze, Tiziano

Belzile, Nelson

Benasciutti, Denis

Benedetti, Matteo

Berdin, Clotilde

Bergmann, Jean-Pierre 
Bernd Schönbauer, Bernd

Berto, Filippo

Beylot, Antoine

Bhole, Sanjeev

Bi, Guijun

Biffi, Carlo Alberto

Billone, Michael

Bizhanov, A. M.

Bland, Leslie

Blázquez, Javier

Blumenau, Marc

Boateng, Joshua S.

Bönisch, Matthias

Bonollo, Franco

Bonora, Pierluigi

Borchers, Christine

Borg, Gregor

Borisenko, Konstantin

Borodachenkova, Marina

Borra, Chenna Rao

Bouguecha, Anas

Boumerzoug, Zakaria

Bowen, Patrick K.

Brahimi, Salim

Brantley, William A.

Brockman, Robert A.

Brown, Andy

Brueckner, Frank

Brundidge, C. L.

Buffa, Gianluca

Burrows, Susan

Busch, Raila

Butt, Javaid

Caballero, Francisca G.

Cabrini, Marina

Calliari, Irene

Campana, Giampaolo

Campanelli, Sabina Luisa

Campbell, John

Cannayen, Igathinathane

Cao, Xinjin

Capela, Carlos

Capelle, Julien
Carabineiro, Sónia A. C.

Carlberg, Torbjorn

Carlone, Pierpaolo

Carpenter, John S.

Carter, Jennifer L. W.

Casalino, Giuseppe

Casari, Daniele

Cavaliere, Pasquale Daniele

Cavalli, Matthew

Cerri, Emanuela

Chalioris, Constantin E.

Chamanfar, Ahmad

Chan, Kwai S.

Chang, Chin-Chun

Chang, Shiying

Chao, Jesus

Charitidis, Costas A.

Chathoth, Suresh Mavila

Chaves, Igor A.

Chavez, Ferman

Chen, Biao

Chen, Bin-Hao

Chen, Chih-Ming

Chen, Hui-Chi

Chen, Shia-Chung

Chen, Shuo

Chen, Tao-Hsing

Chen, Xiaobo

Cheng, Chin-Pao

Chicardi, Ernesto

Chichkov, Boris

Chiu, Tien-Lung

Cho, Jae Ung

Cho, Jae-Hyung

Cho, Ji Ryong

Cho, Young-Rae

Choi, Hyunjoo

Choi, Yoon-Seok

Chou, Kan-Sen

Choudhuri, Deep

Christ, Hans Jürgen

Christen, Philipp

Chuang, Tung-Han
Cicero, Sergio

Citarella, Roberto

Cizdziel, James

Coelho, Luís

Collins, Maurice

Colvin, Robert

Combescure, Alain

Cormier, Denis

Correa, Carlos

Corteen, J.

Couet, Adrien

Cristofolini, Ilaria

Crosky, Alan

Cui, Haitao

Culliton, David

Czerwinski, Frank

Dadbakhsh, Sasan

Dalla Palma, Mauro

Dancette, Sylvain

Das, Goutam

De Lacalle, L. N. López

De Luca, Anthony

De Negri, Serena

De Santis, Michele

DebRoy, Tarasankar

Delaunois, Fabienne

Delogu, Francesco

Delongchamp, Dean

Demers, Vincent

Demir, Ali Goekhan

Demirci, Ibrahim

Denape, Jean

Devesa, Vicenta Angela

Dhakshinamoorthy, Amarajothi

Di Bella, Guido

Di Giovanni, Maria Teresa

Di Schino, Andrea

Diao, Haoyan

Didi, Mohamed Amine

Dietrich, Dagmar

Din, Rameez Ud

Diószegi, Attila

DiSalvo, Francis 
Ditze, André

Dixon, David

Donchev, Alexander

Dorin, Thomas

Dorri Moghadam, Afsaneh

Dosbaeva, Goulnara K.

Drezet, Jean-Marie

$\mathrm{Du}$, Qiang

Duarte, Isabel

Dubourg, Laurent

Duczek, Sascha

Dudziak, Tomasz

Duh, Jenq-Gong

Duprez, Daniel

Dzhurinskiy, Dmitry

Echeverría, Alberto

Eckert, Sven

Efthymiadis, Panos

Eisele, Timothy C.

Eklund, Per

Elahinia, Mohammad

Elisabetta, Gariboldi

Eliyan, Faysal

Eliyan, Faysal Fayez

Engström, Fredrik

Enomoto, Masato

Epishin, Alexander

Erdeniz, Dinc

Ernst, Frank

Esaka, Hisao

Essa, Khamis

Estrin, Yuri

Facchini, Francesco

Fan, Hua-Jun

Farkoosh, Amir

Fatyeyeva, Kateryna

Faulkner, Roy

Fedelich, Bernard

Feliu, Sebastian

Fichtner, Maximilian

Filippov, Lev

Follansbee, Paul

Forcellese, Archimede
Foss, Peter H.

Frage, Nachum

Frantz, Cedric

Fredrickson, Daniel

Freudenberger, Jens

Frulla, Giacomo

Fujii, Hiromichi

Fujita, Satoshi

Galietti, Umberto

Galindo-Nava, Enrique

Gallego, Antolino

Gallino, Isabella

Galtieri, Giovanna

Gamsjäger, Ernst

Gan, Jian

Gancarz, Tomasz

Gangopadhyay, Anup

Garagnani, Gian Luca

Garbovskiy, Yuriy

García, Javier Hidalgo

Garcia-Escorial, Asunción

Garcia-Mateo, Carlos

Gargarella, Piter

Gatterer, Karl

Gaudry, Émilie

Ge, Liya

Gebert, Anett

Gentile, Marialuisa

Gheribi, Aimen.E.

Gherlone, Enrico

Ghidelli, Matteo

Gibmeier, Jens

Gietzelt, Thomas

Gill, Puneet

Giourntas, Lampros

Glinka, Gregory

Goldman, Jeremy

Gong, Pan

Gong, Wu

Gong, Xing

González-Carrasco, José Luis

González-Doncel, Gaspar

Gonzalez-Silveira, Marta
Goodall, Russell

Gostin, Petre-Flaviu

Grabaskas, Dave

Gregory, Haidemenopoulos

Grillo, Federico

Grosdidier, Thierry

Grydin, Olexandr

Guan, Yingchun

Guedes, Aníbal José Reis

Gupta, Rajeev

Gupta, Surojit

Gussone, Joachim

Gust, Ronald

Haddadi, Farid

Hagerman, Michael E.

Haghayeghi, Reza

Haghdadi, Nima

Haider, Waseem

Hallberg, Håkan

Ham, Hyung Chul

Ham, Jong-Oh

Hanaor, Dorian

Hansen, Klaus Schütt

Haque, Rezwanul

Harding, Ian

Harish, Sivasankaran

Harrison, Roger G.

Hassani-Gangaraj, Mostafa

Hattel, Jesper Henri

Hawk, Jeffrey A.

Hayakawa, Tohru

He, Lingfeng

He, Youliang

Heim, Korbinian

Heimann, Robert B.

Hellier, Alan Keith

Henderson, Bill

Hermawan, Hendra

Hernando, Blanca

Herranz, Gemma

Hidalgo-Manrique, Paloma

Higashi, Yuichi

Hirosawa, Shoichi 
Hirsch, Soeren

Hiskey, J. Brent

Ho, Wei-Yu

Ho, Wen-Fu

Hockauf, Kristin

Hofmann, Douglas C.

Hofmeister, Clara

Hohenwarter, Anton

Holappa, Lauri

Hollerweger, Robert

Holtz, Ronald L.

Holuszko, Maria

Holze, Rudolf

Holzweissig, Martin Joachim

Hong, Soon-jik

Hong, Sun Ig

Hort, Norbert

Horvat, Josip

Hsia, Shao-Yi

Hsiang, Hsing-I

Hsiao, Hsu-Liang

Hsieh, Chih-Chun

Hsieh, Pei-Ju

$\mathrm{Hu}$, Xunxiang

Huang, Ke

Huang, Shuo

Huang, Yuanding

Hudgins, Alexander W.

Hufenbach, Julia Kristin

Hug, Eric

Hung, Fei-Yi

Hutchins, David

Hutchinson, Bevis

Hwang, Jinwoo

Hwang, Jiun-Ren

Igari, Toshihide

Infante, Virgínia

Inoue, Hirofumi

Iranzo, Alfredo

Iribarren, José Ignacio,

Ishizaki, Takahiro

Islam, Md. Aminul

Ivanisenko, Julia
Ivanov, Dmitry Sergeevich

Ivey, Douglas

Iwaoka, Hideaki

Izadi, Hossein

Iza-Mendia, Amaia

Jabbari, Mirmasoud

Jacob, Aurélie

Jacquet, Philippe

Jadhav, Niteen

Jaegle, Eric A.

Jajam, Kailash

Jang, Changheui

Jayaprakash, Murugesan

Jha, Kshitij C.

Ji, Vincent

Jiang, Jun

Jiang, Ruinian

Jimenez, Catalina

Jin, Hongmei

Jonsson, Torbjörn

Jothi, Sathiskumar

Jung, Jaepil

Jung, Sung-Mo

Jung, Woo-Gwang

Jyothi, Rajesh Kumar

Kajihara, Masanori

Kakiuchi, Toshifumi

Kalogirou, Orestis

Kamikawa, Naoya

Kamsu-Foguem, Bernard

Kaneko, Kenji

Kaneko, Yoshihisa

Kang, Chun-Gil

Kang, Lijing

Kang, Youn-Bae

Kao, Po-We

Kapranos, Plato

Karaca, Haluk E.

Karjalainen, Pentti

Karlsson, Joakim

Kasada, Ryuta

Kasem, Kasem K.

Kashaev, Nikolai
Kato, Yoshiei

Kaufman, Michael

Kaveh, Edalati

Kawakami, Hiroshi

Kawalla, Rudolf

Kawatra, S. Komar

Kebriaei, Reza

Kecskes, Laszlo

Kelly, Robert G.

Kempen, Karolien

Kendig, Martin W.

Kent, Damon

Khadyko, Mikhail

Khaliq, Abdul

Khan, Tahir

Kharel, Parashu

Kheradmand, Nousha

Khoddam, Shahin

Khun, Nay Win

Kiebach, Ragnar

Kiener, Daniel Kiener

Kim, Donggyu

Kim, Duck Young

Kim, Eunyoung

Kim, Gap-Yong

Kim, Ill-Soo

Kim, Junghwan

Kim, Kyungmok

Kim, Naksoo

Kim, Seon Jin

Kim, Taek-Soo

Kim, Yonghwan

Kim, Yu Chan

Kish, Joey

Kitazono, Koichi

Klöcker, Helmut

Klotz, Ulrich E.

Ko, Ming-Sheng

Ko, Young Gun

Kocaefe, Duygu

Koenigsberger, Erich

Koizumi, Hiroyasu

Kolluri, Murthy 


Komizo, Yu-ichi
Konda Gokuldoss, Prashanth
Kong, Choon Yen
Konosu, Shinji
Koseki, Toshihiko
Kostryzhev, Andrii
Kotoka, Ruben

Koumoulos, Elias P.

Koumousis, Vlasios K.

Kourousis, Kyriakos

Kraemer, Dennis

Krzanowski, James

Kuhn, Bernd

Kukli, Kaupo

Kulkarni, Neha

Kunimine, Takahiro

Kunze, Karsten

Kutschke, Sabine

Kwon, Oh-Kyong

Kwon, Tae-yub

La Mendola, Diego

Laatikainen, Markku

Lafranche, Eric

Lahiri, Abhishek

Laiarinandrasana, Lucien

Lambiase, Francesco

Lampke, Thomas

Lang, Fengqun

Langer, Kristina

Laptev, Alexander

Laracca, Marco

Larouche, Daniel

Lauro, Carlos H.

Lavernia, Enrique

Lavkulich, Les M.

Lay, Sabine

Le, Viet-Duc

LEARDINI, Fabrice

Leclerc, Nicolas

Lee, Chang-Hoon

Lee, Chin C.

Lee, Dong Nyung

Lee, Ho Won
Lee, Jae-Myung

Lee, Jinyi

Lee, Jong-Hyeon

Lee, Jong-Hyun

Lee, Jung G.

Lee, Junghoon

Lee, Kwang Seok

Lee, Min Ku

Lee, Pee-Yew

Lee, Taekyung

Lee, Ying-Chieh

Legrand, Vincent

Lehmusto, Juho

Leidermark, Daniel

Leitner, Martin

Lekakh, Simon

Lekatou, Angeliki

León, Gerardo

Leparoux, Marc

Lertora, Enrico

Lewis, Gladius

Li, Huijun

Li, Jiehua

Liang, Jiandong

Liao, Ying-Chih

Licavoli, Joseph J.

Lieblich, Marcela

Lin, Chao-Sung

Lin, Chun-Ming

Lin, Dan-Jae

Lin, Jau-Wen

Lin, Yuan-Ching

Lincoln, Per

Lindroos, Matti

Lipp, Vladimir

Little, Brenda

Liu, Feng Chao

Liu, Jingjing

Liu, Yi

Llanes, Luis Miguel

Lo Conte, Antonietta

Logé, Roland

López, Antonio Julio
Lostado, Ruben

Louhenkilpi, Seppo

Louzguine, Dmitri

Lozano, Karen

Ludovico, Antonio D.

Ma, Anxin

Ma, Duancheng

Ma, Junjie

Ma, Naiyang

Maass, Robert

Macadre, Arnaud

Madison, Jonathan

Maetz, Jean-Yves

Mahjoub, Reza

Mäkelä, Martin A.

Mamidala, Ramulu

Mandracci, Pietro

Mangipudi, Kodanda

Mankey, Gary

Manukyan, Khachatur V.

Manzoni, Anna M.

Mao, Chuanbin

Mapelli, Carlo

Marco, Coisson

Marinel, Sylvain

Marthinsen, Knut

Martins, José Inácio

Masuda, Yoshitake

Mateo, Antonio

Mathew, Mathew Thoppil

Matsushima, Yuta

Matsuura, Hiroyuki

Matteis, Paolo

Mattern, N.

Maurel-Pantel, Aurélien

Mayer, Herwig R.

Mayer, Thomas

Maziere, Matthieu

McAndrew, Anthony R.

McHugh, Peter

Mcnelley, Terry R.

Melchers, Rob

Melero, Hortensia 
Mendis, Chamini

Meneghetti, Giovanni

Meng, Fanqiang

Meng, Yi

Merkel, Sébastien

Mertens, Anne

Meyers, Marc André

Miani, Fabio

Miao, Jiashi

Milanese, Chiara

Milkereit, Benjamin

Miller, Scott F.

Minakshi, Manickam

Mingler, Bernhard

Miranda, Rosa

Mirihanage, Wajira

Misra, Devesh

Miyajima, Yoji

Miyamoto, Goro

Modigell, Michael

Mohtadi-Bonab, Mohammad Ali

Mokhtari, Omid

Mombelli, Davide

Monchoux, Jean-Philippe

Monetta, Tullio

Mónica, Preciado

Montanari, Roberto

Montgomery, Melanie

Morris, David G.

Mortello, Michelangelo

Moseke, Claus

Mosler, Jörn

Mostafa, Ahmad

Moszner, Frank

Mower, Todd M.

$\mathrm{Mu}$, Wangzhong

Mucha, Jacek

Mughrabi, Haël

Mukherji, Debashis

Müller, Sören

Muñoz, Ángel

Murali, Sarangapani

Murayama, Mitsuhiro
Nagaoka, Toru

Nam, Ki-Woo

Naser, Mohannad

Nazarov, A.

Neves, Filipe

Neves, Rui

Nguyen-Thi, Henri

Nie, Mengyan

Nielsen, Chris Valentin

Nikbin, Kamran

Nobile, Riccardo

O'Dowd, Noel P.

Odeshi, Akindele

Oehring, Michael

Ohshima, Tatsuya

Okayasu, Mitsuhiro

Okitsu, Yoshitaka

Okoro, Sunday Chukwudi

Ola, O. T.

Olivares-Navarrete, René

Oliveira, Fernando

Oliveira, João Pedro

Olowinsky, Alexander

Omrani, Emad

Ono, Kanji

Osawa, Takahito

$\mathrm{Ou}$, Hengan

Owolabi, Gbadebo

Ozel, Tugrul

Palin-Luc, Thierry

Palkowski, Heinz

Pan, Zhiliang

Panagiotis, Spathis

Panagopoulos, C. N.

Pane, Salvador

Panigrahi, Mrutyunjay

Pantazopoulos, George

Pantelakis, Spiros

Pantelis, Nikolakopoulos

Papaiconomou, Nicolas

Papula, Suvi

Paradiso, Valentino

Parbhakar-Fox, Anita
Parisi, Salvatore

Park, Cheol-Min

Park, Eun Soo

Park, Jinman

Park, Nam-Gyu

Park, Sun Hong

Parker, Jonathan

Parthasarathy, Triplicane A.

Pasadas, Dario J.

Pasmanik, Guerman

Patel, Jayesh

Patel, Vikas

Paul, Shiladitya

Pederson, Robert

Pellicer, Eva

Pérez, Pablo

Perron, Aurelien

Petrov, Roumen

Pezzato, Luca

Phaniraj, Madakashira

Picard, Donald

Pickles, Christopher

Pierson, Harry

Pistorius, Petrus Christiaan

Plantevin, Olivier

Plucknett, Kevin P.

Podlaha-Murphy, Elizabeth

Podolskiy, Aleksey V.

Poetschke, Johannes

Pohl, Michael

Pola, Annalisa

Polatidis, Efthymios

Poletti, Marco Gabriele

Porter, David

Potirniche, Gabriel

Pradeep, Konda Gokuldoss

Primig, Sophie

Procaccia, Itamar

Puga, Hélder

Punshon, Tracy

Pyttel, Brita

Qin, Wen

Ragab, Khaled 
Rahimian, Mehdi

Rais-Rohani, Masoud

Ramahi, Omar M.

Ramazani, Ali

Ramin, Aghababaei

Ramous, Emilio

Rams, Joaquín

Rana, Dipak

Rao, Harish

Rao, Kamineni Pitcheswara

Ratvik, Arne Petter

Recarte, Vicente

Reis, Ana

Reny, Pierre

Reverberi, Andrea

Rizzo, Piervincenzo

Roach, Michael D.

Rochford, Luke A.

Rodrigues, Dulce

Rodríguez-Ibabe, José María

Rokni, Reza

Romanoff, Jani

Ronevich, Joseph A.

Ropital, François

Roque, Carla M. C.

Rosas, Omar

Rosato, Antonio

Röttger, Arne

Roy, Christian

Røyset, Jostein

Ruano, Oscar

Rudolph, Jürgen

Ruiz-Bustinza, Iñigo

Runnemalm, Anna

Russell, Alan M.

Sabirov, Ilchat

Sahajwalla, Veena

Saigal, Anil

Sakairi, Masatoshi

Salcedo, Daniel

Salimi-Jazi, Hamid Reza

Salmi, Mika

Sánchez-Amaya, José María
Sandstrom, Rolf

San-José, José T.

Santecchia, Eleonora

Santodonato, Lou

Sarac, Baran

Sasaki, Katsuhiko

Sato, Hisashi

Satriano, Cristina

Sawpan, Moyeenuddin A.

Schneider, Judy

Seetharaman, Sankaranarayanan

Segreto, Tiziana

Semaltianos, N. G.

Semboshi, Satoshi

Senk, Dieter

Senkov, Oleg

Senos, Ana Maria Oliveira Rocha

Seok, Jongwon

Sepúlveda-Escribano, Antonio

Sevostianov, Igor

Shah, Sandeep

Shaha, Sugrib Kumar

Shakur Shahabi, Hamed

Shang, Jianhui

Sharma, Bhupendra

Shen, Zhijian James

Shi, Jianmin

Shi, Zhusheng

Shibata, Etsuro

Shih, Teng-Shih

Shim, Jae-hyeok

Shimamura, Yoshinobu

Shimasaki, S.

Shirvanimoghaddam, Kamyar

Shkirskiy, Viacheslav

Shukla, Pratik

Sillanpaa, Mika

Da Silva, Francisco Gomes

Silvestroni, Laura

Simões, Sónia

Singh, Alok

Singh, Ashish

Singh, Harpal
Singh, Prashant

Slifka, Andrew J.

Smaga, Marek

Smialek, James L.

So, Hongyun

Soleimani Dorcheh, Ali

Soliman, Mohamed

Song, Jun

Song, $\mathrm{Xu}$

Şopu, Daniel

Sorgente, Donato

Sort, Jordi

Sparrow, Graham

Spena, Pasquale Russo

Spiess, Lothar

Srivatsan, Tirumalai S.

Sroka, Marek

Ståhl, Jan-Eric

Stairmand, John

Stanford, Mark

Starke, Peter

Steenari, Britt-Marie

Steer, Julian

Steiner, Matthew

Straumal, Boris B.

Streitenberger, Peter

Stroud, Ian

Stubbins, James F.

Stuhr, Uwe

Sturz, L.

Sugimoto, Koh-Ichi

Suh, Dong-Woo

Sun, I-Wen

Sun, Yufeng

Sun, Yong

Sun, Zhidan

Sunol, Joan Josep

Suryanarayana, Challapalli V.

Svensson, Lars-Erik

Tabuchi, Masaaki

Taha-Tijerina, Jaime

Takacs, Laszlo

Takaichi, Atsushi 
Takezawa, Yusuke

Tang, Chungang

Tang, Fujian

Tang, Zhi

Taruta, Seiichi

Tatlock, Gordon

Tavakoli, Reza

Tavares, Sérgio Manuel Oliveira

Teghil, Roberto

Teixeira, Julien

Telang, Abhishek

Teng, Chong

Tetsuo, Oka

Texier, Damien

Theisen, Werner

Thomas, David J.

Thompson, Steve W.

Thorne, Rebecca J.

Tian, Zhenhua

Tiryakioglu, Murat

Toda-Caraballo, Isaac

Tohgo, Keiichiro

Tomashchuk, Iryna

Tong, Mingming

Toptan, Fatih

Torrens-Serra, Joan

Tourret, Damien

Touzin, Matthieu

Tripathi, Jitendra Kumar

Trueba, Monica

Tsai, Che-Wei

Tsai, Kuo-Ming

Tsao, Lung-Chuan

Tseng, Kuang-Hung

Tsukihashi, Fumitaka

Tsutsumi, Yusuke

Tuncer, Nihan

Tuovinen, Olli H.

Uan, Jun-Yen

Uchtmann, Hermann

Uddin, M. Jasim

Uddin, Mohammad

Ueda, Kyosuke
Uematsu, Yoshihiko

Ueno, Tetsuro

Ueshima, Nobufumi

Ulum, Mokhamad Fakhrul

Ur, Soon-Chul

Uranga, Pello

Ushioda, Kohsaku

Uvarov, Vladimir

Van Der Zwaag, Sybrand

Van Dijk, Niels

Van Hemelrijck, Danny

Van Hooreweder, Brecht

Van Humbeeck, Jan

Van Minnebruggen, Koen

Varez, Alejandro

Varga, Lajos Károly

Varma, Shailendra K.

Vastola, Guglielmo

Vattré, Aurélien

Vedani, Maurizio

Velhinho, Alexandre

Venditti, Iole

Venkata, Kiranmayi Abburi

Ventzke, Volker

Verdeja, Luis Felipe

Veys-Renaux, Delphine

Vidu, Ruxandra

Villa, Elena

Vinogradov, Alexei

Voisey, Katy T.

Vukovic, Sinisa

Vynnycky, Michael

Wagner, Stefan

Wagoner, Robert

Waite, Matthew M.

Walker, John

Walsh, William R.

Walter, Rhys

Wang, Danqi

Wang, De-Yi

Wang, Huei-Sen

Wang, Jinqi

Wang, Jiye
Wang, Junlan

Wang, Yi

Weaver, Mark

Weber, Ludger

Webler, Bryan

Wedd, Rer Quincy

Wei, Dongbin

Weibel, Alicia

Weidner, Anja

Weiss, Sabine

Wen, Haiming

Westermann, Ida

Wiese, Bjoem

Winther, Grethe

Wiskel, J. Barry

Wong, Chee How

Woydt, Mathias

Wright, Wendy

Wu, Shinn-Dar

Wüstefeld, Ch.

Xie, Haibo

Xu, Qiang

$\mathrm{Xu}$, Wei

Yamabe, Junichiro

Yamanaka, Kenta

Yan, Claire Yu

Yanagimoto, Jun

Yandouzi, Mohammed

Yang, Chung-Wei

Yang, Fan

Yang, Ying

Yang, Yo-Lun

Yaron, David

Yeh, Chun-Liang

Yen, Hung-Wei

Yeong, Wai Yee

Yonezawa, Toshio

Yoshida, Sanichiro

Yoshinari, Masao

Yu, Cheng-Ju

$\mathrm{Yu}$, Dawei

Yusenko, Kirill V.

Yvon, Jacques 
Zhang, Chaoqun

Zhang, Guangqing

Zhang, Jianqiang

Zhang, Jie

Zhang, Kuangyuan

Zhang, Lai-Chang
Zhang, Laichang

Zhang, Xiaodan

Zhao, Jingwei

Zhao, Liguo

Zhou, Jie

Zhu, Fan
Zhu, Zhaowu

Zhu, Zhixiong

Ziegler, Donald

Zielinski, Adam

Zucchi, Fabrizio

(C) 2017 by the author; licensee MDPI, Basel, Switzerland. This article is an open access article distributed under the terms and conditions of the Creative Commons Attribution (CC-BY) license (http://creativecommons.org/licenses/by/4.0/). 